dride der Dithiocarbaminsäure aufgefasst werden. Rhodanwasserstoff ist eine ausgesprochene Säure, die Verbindung CSNH, den Senfölen ähnlich, wäre mehr als eine Basis aufzufassen. Die Senföle wirken ja auf conc. Schwefelsäure und Salzsäure ein, unter Bildung von Aminbasen und Kohlenoxysulfid: eine Reaction, welcher wohl eine Verbindung von Senföl mit Säure vorangeht. Man kann daher annehmen, dass bei Gegenwart von Basen, Wasser, Alkoholen, einfachen Aethern und ähnlichen Verbindungen Rhodanwasserstoff die Constitution HSCN hat, dagegen in wasserfreiem Zustande bei Gegenwart von starken Säuren theilweise in der isomeren Form CSNH existirt und zwar mit den Säuren zu Derivaten von Thiocarbaminsäure vereinigt, ähnlich wie dies Leuckart für die Verbindung von Phenylcyanat und Chlorwasserstoff dargethan hat. $\left.{ }^{1}\right)$ Auch habe ich schon angegeben, dass sich wasserfreier Rhodanwasserstoff mit Chlorwasserstoff verbindet. Die Constitution dieser Verbindung wäre dann nach der Analogie $\mathrm{NH}_{2} \mathrm{CSCl}$. Dass sich Schwefelwasserstoff mit diesen Verbindungen leicht in Dithiocarbaminsäure, resp. ihr Anhydrid umsetzen kann, liegt auf der Hand, wie aus folgenden Gleichungen erhellt:

$$
\begin{aligned}
\mathrm{NH}_{2} \mathrm{CSCl}+\mathrm{H}_{2} \mathrm{~S} & =\mathrm{NH}_{2} \mathrm{CSSH}+\mathrm{HCl} \text { oder: } \\
2 \mathrm{NH}_{2} \mathrm{CSCl}+\mathrm{H}_{2} \mathrm{~S} & =\mathrm{NH}_{2} \mathrm{CS} \mathrm{S}+2 \mathrm{HCl} .
\end{aligned}
$$

\title{
Ueber die Darstellung und Eigenschaften des Kohlenoxysulfids;
}

von

\section{Demselben.}

Meine vorstehenden Untersuchungen über die Einwirkung von Säuren auf Rhodanwasserstoffsäure habe ich für die Darstellung des Kohlenoxysulfids nutzbar zu machen gesucht.

1) Vergl. auch Michler: Ber. 8, 1664 nnd Michler und Escherich: Das. 12, 1162 . 
Than, der Entdecker des Kohlenoxysulfids, stellte es dar, einmal durch Synthese aus Kohlenoxyd und Schwefel, sodann aus Rhodanalkalien und Schwefelsäure.

Wie Than selbst angiebt, gewinnt man durch die erste Methode nur eine geringe Ausbeute, und das so erhaltene Kohlenoxysulfid ist mit bedeutenden Mengen von Kohlenoxyd gemischt. Salomon ${ }^{1}$ ) leitet dies Gemisch ron Kohlenoxyd und Kohlenoxysulfid in alkoholischer Kalilauge, wodurch das Bender'sche Salz erhalten wird. Aus diesem ist Kohlenoxysulfid durch Zusatz einer Säure leicht darzustellen. Diese Methode hat aber mancherlei praktische Schwierigkeiten. Zudem hat das so erhaltene Kohlenoxysulfid einen fremdartigen, unangenehmen, der Xanthogensäure ähnlichen Geruch. Besser wäre es, anstatt alkoholischen Kalis, alkoholisches Ammoniak anzuwenden. Thiocarbaminsaures Ammoniak ist nämlich unlöslich in Alkohol und wird von Säuren vollständig in Kohlenoxysulfid und Ammoniak zersetzt. Die angegebenen Methoden haben aber keinen praktischen Werth, da man aus Rhodanalkalien Kohlenoxysulfid leicht und in absoluter Reinheit darstellen kann.

Die Vorschrift von 'Than lautet wie folgt $\left.{ }^{2}\right)$ : „Man trägt in ein erkaltetes Gemisch aus 5 Vol. conc. Schwefelsäure und 4 Vol. Wasser so viel gepulvertes Schwefelcyankalium ein, dass die Masse flüssig bleibt. Die Entwicklung des Gases stellt sich von selbst ein; sollte diese zu stürmisch sein, so kühlt man den Kolben mit kaltem Wasser ab. Sollte dagegen später die Entwicklung nachlassen, so braucht man nur den Kolben mit einer Gaslampe auf einige Augenblicke zu berühren und zeitweise tüchtig zu schütteln. Auf diese Art lässt sich ein constanter und ruhiger Gasstrom erhalten. Da das Gas ausserdem eine Spur von Blausäure, Wasserdampf und Schwefelkohlenstoff enthält, muss man es durch drei U-förmige Röhren leiten, von welchen die erste mit feuchtem Quecksilberoxyd eingeriebene Baumwolle, die zweite in möglichst kleine Splitter zerschnittenen Kautschuk,

\footnotetext{
1) Dies. Journ. [2] 5, 479.

2) Ann. Chem. Suppl. ó, 245. 
die dritte Chlorcalcium enthält. Das so gereinigte Gas wird über Quecksilber gesammelt." - Hofmann hat später den Kautschuk durch eine Aetherlösung von Triäthylphosphin ersetzt.

Wie aus vorstebender Untersuchung hervorgeht, kann die Reaction bei der Einwirkung von Schwefelsäure auf Rhodanwasserstoff verschiedenene Richtungen einschlagen, je nach dem Verhältniss zwischen Rhodanwasserstoff, Wasser und Schwefelsäure. Bei grosser Concentration und keinem oder wenig Ueberschuss von Schwefelsäure wird hauptsächlich Persulfocyansäure gebildet. Ist die Concentration gross und zugleich ein grosser Ueberschuss von Schwefelsäure vorhanden, so werden hauptsächlich Dithiocarbaminsäure und ihre Sulfide gebildet. Nur wenn die Concentration nicht allzugross und zugleich ein grosser Ueberschuss von Schwefelsäure vorhanden ist, wird hauptsächlich Kohlenoxysulfid gebildet.

Es ist nun einleuchtend, dass Than's Methode für die Darstellung des Kohlenoxysulfids nicht zweckmässig sein kann. Das Rhodanalkali muss nicht in fester Form, sondern in Lösung verwendet werden. Die Concentration der Schwefelsäure ist auch grösser, als für die Kohlenoxysulfidbildung zweckmässig ist. Schliesslich hängt der Verlauf der Reaction sehr davon ab, wieviel Rhodanalkali verwendet wird. Setzt man das Rhodanalkali in kleinen Portionen und mit guter Kühlung zu Schwefelsäure von der Concentration, welche Than angiebt, so wird die Masse weiss, dickflüssig und schäumend von auskrystallisirten Sulfiden der Thiocarbaminsäure. Man erhält im Anfange einen kräftigen Strom von Kohlenoxysulfid, der aber bald nachlässt, und im besten Falle wird etwa ${ }^{1 / 4}$ des Rhodanwasserstoffs in Kohlenoxysulfid übergeführt. Setzt man aber soviel Rhodanalkali zu der Säure, dass ein bedeutender Theil davon theils durch das Alkali, theils durch gebildetes Ammoniak neutralisirt wird, so erhält man als Hauptprodukt Persulfocyansäure. In jedem Falle enthält das entwickelte Kohlenoxysulfid, zugleich mit den Körpern, welche Than angiebt, auch $5-10 \%$ Kohlensäure, welche derselbe gänzlich übersehen hat. 


\section{Darstellung von Kohlenoxysulfid.}

Aus einer Reihe von Versuchen, wobei theils das Verhältniss zwischen Schwefelsäure und Rhodanwasserstoff gewechselt hat, theils auch die Concentration der Schwefelsäure verschieden war, hat sich ergeben, dass die Reaction am reinsten nach der Formel $\mathrm{HSCN}+\mathrm{H}_{2} \mathrm{O}=\mathrm{COS}+\mathrm{H}_{3} \mathrm{~N}$ verläuft, wenn $\mathrm{zu}$ einem erkalteten $\mathrm{Gemisch}$ von 290 Ccm. oder 520 Grm. engl. Schwefelsäure und $400 \mathrm{Ccm}$. Wasser $50 \mathrm{Ccm}$. von einer bei gewöhnlicher Temperatur gesättigten Lösung von Rhodanammonium oder Rhodankalium zugesetzt wird. Das Gemisch wird intensiv roth gefärbt, welche Farbe nach kurzer Zeit in eine gelbe übergeht. Bei gewöhnlicher Temperatur wird das Gas sehr langsam entwickelt. Bei $20^{\circ}$ fängt ein regelmässiger Strom von Kohlenoxysulfid an, der bei $23^{\circ}-25^{\circ}$ normale Gerchwindigkeit hat. Schon bei $30^{\circ}$ ist die Gasentwicklung sehr schnell. Man setzt daher das Entwicklungsgefäss in ein grosses Wasserbad von $25^{\circ}$ und hält es bei dieser Temperatur entweder durch ein Gasflämmchen oder dadurch, dass man dann und wann kleine Mengen warmes Wasser in das Wasserbad eingiesst. Zum Schluss, wenn die Gasentwicklung langsamer wird, erhöht man die Temperatur bis auf $30^{\circ}$ und schüttelt wiederholt das Entwicklungsgefäss um. Etwa $1 \mathrm{Grm}$. Rhodanwasserstoff entgeht der Zersetzung. Eine geringe Menge eines gelben Niederschlages wird allmälich gebildet. Dieser ist ein Gemisch von Persulfocyansäure und den Sulfiden der Dithiocarbaminsäure.

Eine bei gewöhnlicher Temperatur gesättigte Lösung von Rhodanammonium hat ein Vol.-Gew. von 1,138 . $50 \mathrm{Ccm}$. davon enthalten 34,58 Grm. Rhodanammonium oder 2b,85 Grm. Rhodanwasserstoff. Eine bei gewöhnlicher Temperatur gesättigte Lösung von Rhodankalium hat ein Vol.-Gew. von 1,42. $50 \mathrm{Ccm}$. davon enthalten 47,92 Grm. Rhodankalium oder 29,11 Grm. Rhodanwasserstoff. Gleiche Volumina gesättigter Lösungen von Rhodanammonium oder Rhodankalium enthalten somit nahe dieselbe Menge Rhodanwasserstoff. Die 
Verhältnisse werden somit dieselben sein, ob nun Rhodankalium oder Rhodanammonium angewendet wird.

Es werden bei Innehaltung von obengenannten Verhältnissen aus $50 \mathrm{Ccm}$. Rhodanammoniumlösung, 34,58 Grm. Rhodanammonium enthaltend, erhalten:

20,0 Grm. Kohlenoxysulfid.

$0,5 \mathrm{Grm}$. Kohlensäure.

0,2 Grm. Dithiocarbaminsäure.

3,0 Grm. Persulfocyansäure und Sulfide der Dithiocarbaminsăture.

0,1 Grm. Schwefelkohlenstoff.

1,0 Grm. unzersetzter Rhodanwasserstoff.

Etwa $75 \%$ des Rhodanwasserstoffs werden somit in Kohlenoxysulfid übergeführt.

Die angewandten Verhältnisse entsprechen 1 Mol. Rhodanammonium + 12 Mol. Schwefelsäure +53 Mol. Wasser. $10 \mathrm{Ccm}$. mehr oder weniger Schwefelsäure, als hier angegeben ist, üben wenig Einfluss auf das Resultat.

Verwendet man eine Schwefelsäure von höherer Concentration als hier angegeben, so enthält das entwickelte Kohlenoxysulfid mehr Kohlensäure und in Folge dessen wird auch mehr von Sulfiden der Thiocarbaminsäure gebildet. Durch gesteigerte Concentration der Schwefelsäure kann der Kohlensäuregehalt des Kohlenoxysulfids bis zu 20\% erhöht werden. Erniedrigt man die angewendete Menge von Schwefelsäure unter Beibehaltung derselben Concentration, so bleibt ein grosser Theil des Rhodanwasserstoffs intakt. Wird aber die Menge der Schwefelsäure erniedrigt, die Concentration dagegen erhöht, so geht ein grosser Theil des Rhodanwasserstoffs in Persulfocyansäure über.

Salzsäure kann natürlich auch bei der Darstellung des Kohlenoxysulfids verwendet werden, aber wie es scheint nicht mit demselben Vortheil. Es ist mir nicht geglückt, eine Concentration der Salzsäure ausfindig zu machen, welche eine so vollständige Ueberführung des Rhodanwasserstoffs in Kohlenoxysulfid hervorbringt wie mit Schwefelsäure. Die Reaction scheint immer zum grossen Theil gleichzeitig nach den anderen schon angegebenen Richtungen zu verlaufen. 
Reinigung des Kohlenoxysulfids.

Wie gesagt, enthält das entwickelte Kohlenoxysulfid als Verunreinigungen kleine Mengen von Schwefelkohlenstoff, mehr oder weniger Kohlensäure, Feuchtigkeit und Spuren von Rhodanwasserstoff.

A. W. Hofmann, der Entdecker der Phosphine, hat auch die starke Verwandtschaft zwischen Schwefelkohlenstoff und den Phosphinen kennen gelehrt. Bekanntlich verbinden sich die beiden Körper zu einem schön roth gefärbten, krystallisirten Körper. Er reinigte das Kohlenoxysulfid vom Schwefelkohlenstoff dadurch, dass er das Gas durch eine ätherische Triäthylphosphinlösung leitete. „Nachdem das Gas einige Zeit durch die mit Triäthylphosphin getränkte Baumwolle getrieben war, hatten sich die dem Eintritt desselben zunächst liegenden Schichten intensiv roth gefärbt; diese Färbung wurde in den weiter liegenden Schichten schwächer und schwächer; über die Mitte hinaus war sie nicht mehr wahrnehmbar."

Bei meinem Versuche über das Absorptionsvermögen des Triäthylphosphins für Schwefelkohlenstoff habe ich wahrgenommen, dass dieses höchst bedeutend abnimmt mit zunehmender Verdünnung des Phosphins sowohl mit Aether wie mit Alkohol. Das reine unverdünnte Phosphin absorbirt Schwefelkohlenstoff mit grosser Energie. Bei gemässigtem Strom ist die Absorption in der That vollständig, wenn das Gas erst eine kleine Flasche oder ein Knierohr mit höchstens 1 bis $2 \mathrm{Ccm}$. Phosphin passirt. Die Vollständigkeit der Absorption kann dadurch leicht gezeigt werden, dass das Phosphin in einer zweiten ähnlichen Flasche nicht roth gefärbt wird. Das ausgetretene Gas bekommt aber den eigenthümlichen Geruch der Phosphine. Dieser kann weggenommen werden und das Kohlenoxysulfid zugleich trocken erhalten werden dadurch, dass das Gas durch concentrirte reine Schwefelsäure geht. Gewöhnliche englische Schwefelsäure ist dazu nicht zweckmässig. Sie wird dadurch etwas missfarbig und das ausgetretene Kohlenoxysulfid enthält Spuren eines Gases, 
welches Jodstärke entfärbt. Reine Schwefelsäure hält sich dagegen völlig klar, und das ausgetretene Gas enthält weder Spuren von schwefliger Säure noch von Schwefelwasserstoff, auch nachdem Kohlenoxysulfid Tage lang in Berührung mit der Säure gewesen ist. Es mag weiter erwähnt werden, dass unverdünntes Phosphin auch in der Beziehung vortheilhaft ist, dass das Kohlenoxysulfid dabei nicht mit Aetherdämpfen verunreinigt wird.

Es liegt auf der Hand, dass nur Alkalien die Kohlensäure von dem Kohlenoxysulfid trennen können. Ueber das Verhalten des Kohlenoxysulfids zu Alkalien führt Than folgendes an: „Kaliumhydrat, sowie alkalische Metallhydrate überhaupt, absorbiren das Gas etwas langsamer, aber ebenso vollständig wie die Kohlensäure." Ich nahm aus diesem Grunde an. dass Kalilauge kaum angewendet werden konnte zur Trennung von Kohlensäure und Kohlenoxysulfid. Der Versuch lehrte aber, dass dies keineswegs der Fall ist. Im Gegentheil, Kalilauge von der Concentration, welche angewendet wird bei der Absorption von Kohlensäure bei Verbrennungsanalysen ( 1 Thl. Kalihydrat auf 2 Thle. Wasser), war rollständig zweckmässig. $100 \mathrm{Grm}$. Kalilauge von obengenannter Concentration absorbirten pro Stunde nur 0,1-0,2 Grm. Kohlenoxysulfid, wenn dieses Gas Blase für Blase die Kalilauge passirt. Wird das entwickelte Kohlenoxysulfid durch eine $20 \mathrm{Cm}$. hohe Schicht von Kalilauge geleitet, so ist bei mässigem Strom die Absorption von Kohlensäure vollständig, während von dem Kohlenoxysulfid nur etwa $7 \%$ absorbirt werden. ${ }^{1}$ )

Unter gewöhnlichen Verhältnissen ist es somit zur Reinigung des Kohlenoxysulfids nur nöthig, das Gas durch Kalilauge zu leiten. Absolut rein erhält man das Gas, wenn es erst durch Kalilauge, nachher durch Triäthylphosphin und zuletzt durch conc. reine Schwefelsäure geleitet wird.

1) Uebrigens hat schon Cl. Winkler in seiner ,Anleitung zur qualitativen Gasanalyse" S. 112 auf die geringe Absorption des Kohlenoxysulfids durch Kalilauge aufmerksam gemacht.

E. v. $M$. 


\section{Analytische Trennung und Bestimmung des Kohlenoxysulfids.}

Wie oben erwähnt, absorbirt Kalilauge äusserst langsam das Kohlenoxysulfid. Kalilauge kann somit nicht angewendet werden bei der quantitativen Bestimmung desselben. Alkoholische Kalilauge absorbirt auch Kohlenoxysulfid viel zu langsam. Berthelot ${ }^{1}$ ) führt an, dass festes, mit Alkohol befeuchtetes Kali das Gas vollständig absorbirt. Dasselbe habe ich auch gefunden, aber das Absorptionsvermögen nimmt sehr bald ab. Alkalische Blei- und ammoniakalische Silberlösung absorbiren auch das Gas sehr langsam. Folgendes Absorptionsmittel für Kohlenoxysulfid habe ich dagegen zweckmässig gefunden. Concentrirte Kalilösung (1 Thl. Kali und 2 Thle. Wasser) wird mit ihrem gleichen Volum Alkohol gemischt. Hierdurch wird Kohlenoxysulfid rollständig, wie geschwind auch der Gasstrom ist, absorbirt. In der Lösung setzt sich hierbei keine krystallisirte Substanz ab.

Hat man ein Gemisch von Gasen, in welchen Kohlenoxysulfid bestimmt werden soll, so wird das Gasgemisch somit erst durch Kalilauge in langsamem Strom durchgeltitet. Hierdurch werden alle Gase von saurem Charakter entfernt. Hiernach wird das Gas durch Triäthylphosphin geleitet, um Schwefelkohlenstoff $\mathrm{zu}$ entfernen. Dann folgt das obengenannte Absorptionsmittel für Kohlenoxysulfid. Die Gewichtsvermehrung rührt ausschliesslich von diesem Gase her. Etwas von dem Kohlenoxysulfid wird aber auch von der Kalilauge absorbirt und findet sich dessen Schwefelgehalt in der Lauge als Alkalisulfide, resp. unterschwefligsaures Alkali. In einer besonderen Probe wird der Gehalt des Gasgemisches an Schwefelwasserstoff bestimmt durch eine mit Schwefelsäure stark sauer gemachte Kupfervitriollösung. Diese zersetzt nämlich nicht das Kohlenoxysulfid. Geht etwas unabsorbirt durch den ganzen Apparat, so gehört es zu den indifferenten Gasarten und wird durch die gewöhnliche gasanalytische Methode bestimmt.

I) JB. 4, 1880. S. 160. 
Ich habe auch untersucht, ob das in genannter Weise entwickelte Kohlenoxysulfid Cyanwasserstoff enthält, was aber nicht der Fall war. $\mathrm{Zu}$ diesem $\mathrm{Zwecke}$ wurde das Kohlenoxysulfid, nachdem zuerst der Schwefelkohlenstoff entfernt war, durch alkoholisches Ammoniak absorbirt. Es wurden dabei prachtvolle Krystalle von monothiocarbaminsaurem Ammoniak gebildet, und die Absorption des Kohlenoxysulfids war vollständig. Bei einem Gehalt des Kohlenoxysulfids an Cyanwasserstoff sollte dieses sich als Cyanammonium in der Lösung finden. Nachdem die Krystalle von thiocarbaminsaurem Ammoniak entfernt und die Lösung durch Salpetersäure sauer gemacht war, gab Silbernitrat keine Spur eines Niederschlages.

\section{Die Eigenschaften und Reactionen des Kohlen- oxysulfids.}

Da das Kohlenoxysulfid, welches Than dargestellt hatte durch Schwefelkohlenstoff und namentlich durch Kohlensäure verunreinigt war, so hat Than natürlich die Eigenschaften und Reactionen des Kohlenoxysulfids nicht richtig angeben können.

Von dem Geruch des Gases erwähnt derselbe: „Das Gas hat einen der Kohlensäure nicht unähnlichen Geruch, der zugleich aromatisch an Harze und gewissermassen an Schwefelwasserstoff erinnert, aber nicht unangenehm ist." Man erkennt leicht, dass dies auch für ein Gemisch von Kohlensäure und Schwefelkohlenstoff gültig ist. Das reine Kohlenoxysulfid ist vollständig geruchlos. Fine ganz frische wässrige Lösung von Kohlenoxysulfid ist auch völlig geschmacklos und nicht süsslich wie Than angiebt. Das Kohlenoxysulfid in reinem Zustande ist also sowohl ohne Geschmack wie ohne Geruch.

Die Einwirkung des Gases auf das Nervensystem ist höchst merkwürdig und nahe übereinstimmend mit dem des Stickoxyduls. Wird reines Kohlenoxysulfid eingeathmet, so bemerkt man in den ersten 10 Secunden nicht das Geringste. Ylötzlich stellt sich Schwindel ein. Man kann nicht 
aufrecht stehen, ohne sich zu stützen. Ein eigenthümliches Gefühl von Beklemmung der Brust und Ohrensausen stellt sich ein. Wenn das Einathmen nicht fortgesetzt wird, so dauern diese Symptome höchstens 2 Minuten, nach welcher Zeit sie eben so plötzlich verschwinden, wie sie gekommen waren, ohne Spuren von Kopfschmerzen oder andere Unannehmlichkeiten zu hinterlassen.

Wird das reine Gas durch eine bei gewöhnlicher Temperatur gesättigte Lösung von Barythydrat geleitet, so dauert es wenigstens eine halbe Minute, ehe man eine Trübung bemerken kann. Man hat hier eine Reaction, wodurch es möglich ist, auch sehr kleine Mengen von Kohlensäure qualitativ in dem Kohlenoxysulfid nachzuweisen. Wird z. B. gewöhnliche Luft durch Barythydrat geleitet, so stellt sich eine sichtbare Trübung ein, lange bevor noch eine halbe Minute verlaufen ist.

Wird das reine Gas durch eine klare Lösung von essigsaurem Bleioxyd geleitet, so dauert es mindestens $1 / 4$ Stunde, ehe man die geringste Trübung in der Bleizuckerlösung bemerkt. Nach Verlauf dieser Zeit wird die Lösung allmälich missfarbig. Der Niederschlag wird dunkler und dunkler. Diese Reaction ist sehr charakteristisch für Kohlenoxysulfid und.kann auch benutzt werden, um äusserst geringe Spuren von Schwefelwasserstoff in dem Gase qualitativ anzuzeigen. Auch wenn der Gehalt von Schwefelwasserstoff äusserst gering ist, so lässt sich doch lange vor dem Verlaufe einer $1 / 4$ Stunde eine schwarze Trübung in der Bleiacetallösung bemerken.

Das empfindlichste Reagens auf reines Kohlenoxysulfid ist Jodstärke. Wenn durch eine mit einer. Spur Jod klar blau gefärbte Stärkelösung reines Kohlenoxysulfid durchgeleitet wird, so dauert es bei gewöhnlicher Temperatur mindestens 8 Minuten, ehe man eine Abnahme in der Intensität der Farbe beobachten kann. Während der nächsten 10 Minuten beobachtet man sebr gut, dass die Farbe erst in Violett, nachher in Roth übergeht. Schliesslich verschwindet sie vollständig. Diese Reaction setzt voraus, dass keine anderen auf die Jodlösung einwirkende Substanzen 


\section{Klason: Ueber die quantitative Bestimmung}

anwesend sind. Die Reaction zeigt auch, dass Kohlenoxysulfid nur langsam Wasser aufnimmt unter Bildung von Kohlensäure und Schwefelwasserstoff. Dieser Verlauf kann durch Jodstärke leicht quantitativ verfolgt werden.

In stark sauren Lösungen tritt die Hydration des Kohlenoxysulfides gar nicht ein. In Berührung mit reiner, conc. Schwefelsäure sowohl, wie mit halbverdünnter wird das Kohlenoxysulfid gar nicht zersetzt. Will man daher Kohlenoxysulfid in Gasform auffangen, so wird dazu zweckmässig anstatt Quesksilber ein Gemisch von gleichen Volumen conc. reiner Schwefelsäure und Wasser benutzt. Da dieses Ge misch die Haut wenig angreift, so kann man ebenso bequem mit Kohlenoxysulfid wie mit Gasen, die über Wasser aufgesammelt werden können, operiren.

\section{Ueber die quantitative Bestimmung des Rhodanwasserstoffs; \\ von}

\section{Demselben.}

Rhodanwasserstoff und Salzsäure verhalten sich zum Silbersalz in ganz derselben Weise. Das Ausfallen des Rhodansilbers tritt unmittelbar vor der Vollständigkeit der Fällung ein, und der Endpunkt erscheint mit derselben Deutlichkeit wie mit Salzsäure. Man kann daher im Voraus annehmen, dass dieselben Bestimmungsmethoden, die für Salzsäure gültig sind, auch für den Rhodanwasserstoff angewendet werden können. Angestellte Versuche zeigten, dass dies der Fall ist.

Die Titrirung der Salzsäure mit Silbersalz und neutralem Kaliumchromat als Indicator ist zu wohl bekannt, um hier näher erwähnt zu werden. Sie setzt bekanntlich voraus, dass die Lösungen neutral sind oder doch nur eine Spur Alkalicarbonat (nicht Bicarbonat) im Ueberschuss enthalten. 